\title{
PARCERIAS PARA A GESTÃo DO USO PÚBLICO EM PARQUES
}

\section{Douglas de Souza Pimentel ${ }^{1}$}

\section{Resumo}

Os parques são icônicos na discussão sobre a conservação da biodiversidade aliada a visitação dessas áreas protegidas. O presente artigo tem por objetivo discutir os conceitos e atividades relacionadas ao uso público em unidades de conservação (UCs). O uso público é conceituado como o uso indireto dos recursos ambientais nas diferentes formas de visitação de uma UC e, portanto, deve sempre ser orientado pelos preceitos da sustentabilidade e mitigação de impactos. Assim, a pesquisa científica, a educação e interpretação ambiental, a recreação em contato com a natureza, bem como o turismo ecológico são classificados como uso público. Porém essas atividades causam impactos ambientais negativos, que devem ser monitorados e manejados. O Grupo de Estudos Interdisciplinares da UERJ vem estabelecendo parcerias com o Museu de Arqueologia de Itaipú, o Parque Estadual da Serra da Tiririca e o Parque Nacional da Serra dos Órgãos para o desenvolvimento de atividades de ensino e pesquisa com o intuito de fomentar a educação ambiental e o monitoramento de impactos. Frente aos conceitos e experiências apresentadas o artigo aponta as vantagens do estabelecimento de parcerias entre os gestores do uso público de parques e a universidade.

Palavras chave: Uso público; arques; colaboração; interdisciplinaridade; manejo.

\begin{abstract}
The parks are iconic in the discussion of biodiversity conservation combined with visitation of these protected areas. This article aims to discuss the concepts and activities related to public use in protected areas. Public use is conceptualized as the indirect use of environmental resources considering the different forms of protected areas' visitation and therefore, should always be guided by the principles of sustainability and impact's mitigation. Thus, scientific research, environmental education and interpretation, recreation, contact with nature and eco-tourism are classified as public use. But these activities cause negative environmental impacts, which should be monitored and managed. The Group for Interdisciplinary Studies of UERJ has established partnerships with the Museum of Archaeology of Itaipu, the Serra da Tiririca State Park and the Serra dos Órgãos National Park to the development of teaching and research with the goal of promoting environmental education and impact monitoring. Facing the concepts and experiences presented the article points out the advantages of partnerships between public use's managers of parks and university.
\end{abstract}

Keywords: Public use; parks; collaboration; interdisciplinary; management

\section{Introdução}

Os parques são icônicos nas discussões sobre conservação da biodiversidade, no Brasil e no mundo. Os ecólogos geralmente veem essas áreas protegidas como uma solução para viabilizar a preservação e manejo de ecossistemas. No entanto, os parques estão imersos em um ambiente político e institucional, bem como assumem diferentes representações para a sociedade (PIMENTEL; MAGRO, 2011). Nesse sentido, raramente são uma solução

\footnotetext{
${ }^{1}$ Professor Adjunto da Universidade do Estado do Rio de Janeiro e da Universidade Federal Fluminense. Coordenador do Grupo de Estudos Interdisciplinares do Ambiente (GEIA) - E-mail: douglasgeia@ gmail.com
} 
consensual, o que amplifica os problemas de sua administração, que tem que lidar com todo o espectro de atividades relativas à conservação - das questões ecológicas à relação do parque com as pessoas, sejam seus visitantes ou a população local.

Para tal, há a necessidade de um grande investimento estatal, mas o quadro geralmente observado no ambiente de gestão dessas unidades de conservação não reflete essa demanda. Há carência de investimentos e pessoal que dificultam o alcance dos objetivos conservacionistas. Nesse âmbito, a atuação das instituições universitárias nos parques é relevante para geração de dados sobre a ecologia e biodiversidade, mas também há uma grande responsabilidade socioambiental com a geração e divulgação desse conhecimento.

Considerando um passo adiante, cabe o questionamento se os laços entre os gestores dessas unidades de conservação e os pesquisadores podem ser estreitados. Para tal, esses últimos precisam entender que o seu trabalho nas áreas naturais é importante, porém não suficiente para que efetivamente ocorra a conservação, pois os ecossistemas, que muitas vezes estão abrigados em unidades de conservação, não estão apartados das influências e impactos humanos. Por sua vez, os primeiros também tem que assumir uma postura proativa para driblar as carências com que tem que lidar diariamente. Nesse sentido, as questões sobre o uso público dos parques podem representar um ponto de congruência nessa relação, pois estão relacionadas ao manejo socioambiental da unidade de conservação, envolvendo aspectos que transcendem os limites da mesma e podem auxiliar no ganho de significado da conservação para a sociedade.

O presente artigo tem por objetivo discutir os conceitos e atividades relacionadas ao uso público em unidades de conservação, sintetizando as vantagens da aproximação entre a sua gestão, das ações universitárias de ensino, pesquisa e extensão.

\section{O que é uso público?}

Para o entendimento do que significa o termo uso público, uma história precisa ser contada. Essa começa em 1872, com a criação do Parque Nacional de Yellowstone nos Estados Unidos da América. A ideia por trás da instituição do primeiro parque nacional no mundo era reservar um lugar de grande beleza cênica para visitação por pessoas de uma sociedade em crescente processo de urbanização, de consolidação do capitalismo e de fronteiras (ABAKERLI, 2001; DIEGUES, 2004). Assim, mesmo considerando que já havia registros na história mundial de reservas de áreas por motivos religiosos ou para a restrição do uso dos recursos naturais pela população em geral, como madeira e caça, pode-se dizer que um novo argumento para criar áreas protegidas estava surgindo: a preservação da natureza frente ao reconhecimento do poder da espécie humana em transformar as paisagens (BENSUSAN, 2006).

Logo, os parques também passariam a representar uma maneira em que muitas pessoas vivenciam a natureza, assumindo esse referencial, no gradiente em que a sociedade urbana encontra-se no outro extremo, do espaço tecnologicamente transformado (WATSON, 2000; WATSON; LANDRES, 1999). Essa nova percepção sobre a natureza, "abrigada" em parques, sustenta até hoje as bases científicas da conservação ambiental (GODOY, 2006). 
No mesmo sentido, esse modelo emergente de conservação forneceu as bases de uma racionalização politicamente viável para a reserva de áreas protegidas. O turismo poderia sustentá-las conceitual e economicamente. West, Igoe e Brockington (2006) consideram que a criação de Yellowstone instaurou um modelo de conservação baseado no "consumo" de paisagens e experiências genéricas dessas áreas protegidas, encaradas como produtos. Essa pode ser uma opinião radical, mas de fato, as atividades de visitação nos primeiros parques foram muito incentivadas, inclusive com a contratação de profissionais de propaganda para convencer as elites que a viagem era segura (ABAKERLI, 2001; WEST; IGOE; BROCKINGTON, 2006).

Considerando ainda Yellowstone, o parque foi um dos principais promotores do grande apreço do público norte americano pela visitação de áreas naturais protegidas. Os turistas usavam veículos puxados por cavalos em estradas acidentadas para chegar ao local, no início do século XX; em 1903, o presidente Theodore Roosevelt, entusiasta de aventuras em ambientes naturais, é fotografado no Parque; em 1931 é desenvolvido o modelo White de ônibus de turismo, com capota dobrável, especialmente para a sua visitação (NPS, 2013). Em 1958 foi criado o personagem Zé Colmeia, que passa a fazer grande sucesso junto às crianças, com as suas peripécias no Parque de "Jellystone", em referência ao parque real. Frente a todo esse investimento e propaganda, a visitação em parques passa a constituir um forte traço cultural dos EUA, ainda observado nos dias de hoje e esse modelo de conservação racionalizado pelo turismo espalha-se pelo mundo.

No Brasil, os ecos de todo esse movimento reverberaram no engenheiro André Rebouças, ainda em 1876. Ele propôs então a criação dos Parques Nacionais de Sete Quedas (PR) e Bananal (GO) (BENSUSAN, 2006). Porém, somente em 1937 é instituído o Parque Nacional de Itatiaia, no Estado do Rio de Janeiro. Gradativamente, outros parques e áreas protegidas foram sendo criadas e, ao longo de muitos anos, foi aumentando a necessidade de uma sistematização de categorias e objetivos, o que finalmente é estabelecida em 2000, com o Sistema Nacional de Unidades de Conservação (SNUC - Lei no 9985 de 18 de julho de 2000). No que concerne aos parques, o art. 11 dessa lei estabelece que esses (BRASIL 2002. p.16, grifos do autor):

[...] têm como objetivo básico a preservação de ecossistemas naturais de grande relevância ecológica e beleza cênica, possibilitando a realização de pesquisas científicas e o desenvolvimento de atividades de educação e interpretação ambiental, de recreação em contato com a natureza e de turismo ecológico.

Interessante notar que a lei traz referência à beleza cênica de um sítio como condição para a instituição de um parque, reafirmando a sua histórica destinação para o turismo.

O SNUC também estabelece o conceito de conservação, relacionando-o ao uso sustentável dos recursos naturais frente à necessidade de garantir a manutenção da biodiversidade. Assim, várias estratégias, como a preservação e recuperação ambiental podem ser adotadas. Logo, esse uso dos recursos tem que ser manejado e para a definição legal dos procedimentos de manejo, os parques estão alocados na categoria de proteção integral, definida pelo objetivo primordial de preservação da natureza. Nesse sentido, apenas o uso 
indireto dos recursos ambientais é permitida. Esse não pode envolver o "[...] consumo, coleta, dano ou destruição dos recursos naturais" (BRASIL, 2002, p.6). Porém, as atividades de visitação podem trazer uma série de impactos negativos à área protegida e, portanto, mesmo essa forma de uso deve obedecer a diferentes preceitos de sustentabilidade. Dessa forma, o uso sustentável é definido pela mesma lei, no inciso XI do artigo segundo (BRASIL, 2002, p.6) como a "exploração do ambiente de maneira a garantir a perenidade dos recursos ambientais renováveis e dos processos ecológicos, mantendo a biodiversidade e os demais atributos ecológicos de forma socialmente justa e economicamente viável”.

Logo, há uma preocupação obvia com o estabelecimento de mecanismos de controle de impactos decorrentes da visitação nos parques, tanto pela obediência a lei e esforços que focam na conservação ambiental, quanto para evitar a perda do seu valor estético, principal atrativo da crescente visitação nessa categoria de unidade de conservação (MAGRO, 2003). Assim, o termo uso público é adotado pelos órgãos oficiais brasileiros ligados ao seu manejo. Esse pode ser definido como o usufruto gozado pelo público usuário, que realiza diferentes atividades como a recreação, o turismo, a educação ambiental, a pesquisa e até mesmo, ritos religiosos (MAGRO, 1999). Nesse sentido, o uso público pode ser conceituado como o uso indireto dos recursos ambientais que se dá sob as diferentes formas de visitação de uma unidade de conservação e, portanto, deve sempre ser orientado pelos preceitos da sustentabilidade e mitigação de seus impactos.

\section{A visitação nas unidades de conservação}

Considerando primeiro as unidades de conservação de uso sustentável, o termo uso público pode ser aplicado e também deve ser instituída uma política relacionada à visitação. É crescente a discussão do turismo de base comunitária, bem aplicado a essa categoria de manejo, pois conceitualmente, essa forma de atividade turística foca na ética das relações sociais e valorização dos recursos ambientais locais (ARAÚJO; GELBCKE, 2008). Esses preceitos estão de acordo com os objetivos de promoção do desenvolvimento sustentável e valoração econômica e social da biodiversidade, instituídos pelo SNUC. Além disso, para que o turismo de base comunitária seja viabilizado, há a necessidade do desenvolvimento da organização democrática da população receptora, o que pode se estender para os conselhos gestores das unidades de conservação, ampliando a participação comunitária na gestão do seu patrimônio ambiental.

Para aquelas de uso mais restrito, como as estações ecológicas e as reservas biológicas, a educação ambiental e a pesquisa podem acontecer, mediante condições especiais e autorização. Porém, ainda considerando as unidades de conservação de proteção integral, os parques têm como objetivo primordial, além da preservação da natureza, abarcar todas as formas de uso público. Portanto, as questões relacionadas à sua visitação são muitas vezes tratadas como prioritárias. Mas esse aspecto não se traduz necessariamente em investimentos para o manejo dessas atividades. Dessa maneira, há necessidade de projetos e políticas que fomentem uma forma de uso público, mais direcionada e planejada, que permita o desenvolvimento do ecoturismo e da pesquisa (PIMENTEL; MAGRO, 2013). 
A recreação, por sua vez, caracteriza-se pelas atividades desenvolvidas em momentos de lazer (TAKAHASHI, 2004) e não representa necessariamente uma atividade turística nas unidades de conservação e sim de visitação, pois é importante considerar também aquelas áreas protegidas que estão dentro ou próximas dos centros urbanos e, portanto, podem ser facilmente acessadas. A educação ambiental, por sua vez, é um eixo integrador do uso público, pois representa uma atividade prevista pela lei do SNUC para todas as unidades de conservação (PIMENTEL, MAGRO, 2013).

\section{A pesquisa científica}

Quando se fala em pesquisa em unidades de conservação, principalmente nas de proteção integral, geralmente se pensa na prospecção da biodiversidade e no entendimento dos processos ecológicos. Realmente, as estações ecológicas e as reservas biológicas foram pensadas nesses termos, objetivando a preservação e o ganho de conhecimentos biológicos e ecológicos de populações e ecossistemas sem grande interferência humana. Mas a pesquisa e educação são as formas especiais de uso público nessas categorias de manejo e somente são permitidas mediante autorização especial concedida pelos órgãos gestores. No âmbito federal, agora considerando todas as unidades de conservação, o licenciamento dessas atividades encontra-se bem estruturado pelo Sistema de Autorização e Informação em Biodiversidade SISBIO (BRASIL, 2007).

As atividades de uso público tem o objetivo de despertar no visitante novos valores e comportamentos, almejando também a consolidação do SNUC (TAKAHASHI, 2004). Assim, coloca-se em evidência a importância da pesquisa sobre o uso público em parques brasileiros, tanto para a estruturação dos processos de monitoramento de impactos positivos e negativos da visitação, que inclusive devem ser adequados à diversidade e particularidades dos biomas nacionais, quanto para se avaliar a efetividade da visita na promoção dessa nova percepção sobre as questões ambientais. Porém, no âmbito acadêmico foca-se na pesquisa básica. No administrativo, infelizmente há poucos investimentos na produção desse conhecimento.

\section{O ecoturismo}

O conceito de ecoturismo o define como uma viagem a áreas naturais, compromissada com a conservação ambiental e com as culturas locais (TAKAHASHI, 2004; WESTERN, 1999). Como os parques assumem a representação social da natureza e possuem os atributos estéticos para atrair o público ecoturista, verifica-se que a visitação dessas unidades de conservação vem crescendo a cada ano e esse fato pode afetar a definição dessa vertente do turismo.

Segundo Boo (1999), os impactos negativos do ecoturismo referem-se a potencial degradação ambiental, injustiças sociais, instabilidades econômicas locais e mudanças socioculturais negativas. Os positivos relacionam-se a geração de receita, a criação de empregos e a promoção da educação ambiental. Takahashi (2004) alerta ainda que as atividades ecoturísticas encontram-se desordenadas e direcionadas meramente pelos interesses mercadológicos, o que comprometeria o alcance dos objetivos socioeconômicos e ambientais. Considerando que os parques e outras unidades de conservação são os receptores lógicos do 
ecoturismo, ratifica-se que há uma conceitual ligação com a sustentabilidade do uso público nessas áreas protegidas. Assim, essas atividades devem envolver o planejamento, gestão e monitoramento de impactos. Pode-se dizer então que sem esse grande esforço e investimento na sua administração, dificilmente se alcança plenamente o ecoturismo.

\section{O lazer e a recreação}

O lazer é comumente adotado para atividades esportivas, recreativas ou culturais e melhor conceituado, segundo Takahashi (2004) como uma noção de tempo disponível para a recreação. Essa, por sua vez estaria relacionada às atividades de diversão, sendo esse o termo técnico adotado pelos especialistas em visitação de áreas protegidas no âmbito internacional (MAGRO, 1999). A recreação que envolve o contato com a natureza pode trazer benefícios psicológicos e mudanças comportamentais importantes para a conservação ambiental e cultural (TAKAHASHI, 2004).

A diferença entre o ecoturismo e a recreação em ambientes naturais reside no tempo de deslocamento e permanência nos destinos visitados. Além disso, o ecoturismo engloba conceitualmente a responsabilidade com a conservação ambiental e o que define essa observância para a recreação é quando essa é realizada nos parques, cujos gestores devem preocupar-se com os impactos negativos decorrentes de ambas as atividades. Porém, as duas formas de visitação também podem impactar positivamente a área protegida, ao catalisar uma reflexão mais profunda sobre os valores individuais no sentido de fomentar mudanças comportamentais ambientalmente responsáveis, conciliando a demanda e a satisfação do usuário com a conservação da área (SILVA; COSTA NETO, 2007).

\section{A educação e interpretação ambiental}

A educação ambiental é caracterizada como um processo que busca a compreensão crítica das interações entre a sociedade e o ambiente (DIAS, 2003; UNESCO, 1998). No Brasil, o termo consolidou-se com a Constituição Federal de 1988 e com o Programa Nacional de Educação Ambiental (PRONEA) de 1994. Em 1999 é publicada a lei que estabelece a promoção da educação ambiental como uma política pública, a Política Nacional de Educação Ambiental (PNEA - LEI No 9.795 de 27 de abril de 1999) que reforça a visão desse processo educativo acontecendo de forma articulada em espaços formais e não formais de ensino (LOUREIRO, 2004; PIMENTEL; MAGRO, 2013). No entanto, a regulamentação dessa lei somente acontece em 2002, quando é definido o órgão gestor da PNEA, que depende da articulação e a troca de informações balizadas por pesquisa socioambiental entre os Ministérios da Educação e do Meio Ambiente, para a tomada de decisões. Porém essa dinâmica ainda não é muito eficiente (VASCONCELLOS, 2006).

No mesmo sentido, Pimentel e Magro (2013. p.45) avaliam que:

[...] os Parques podem servir como lócus das ações de consolidação da Política de Educação Ambiental, pois: (1) representam um eixo básico estrutural de integração entre as ações do Ministério do Meio Ambiente e Ministério da Educação; (2) têm como premissa básica o uso público qualificado pela aquisição de conhecimentos e habilidades, 
reaproximando as pessoas dos ambientes naturais pela afetividade e reflexão sobre como suas ações o afetam; (3) permitem uma visão prática e crítica das relações da sociedade com a natureza de uma maneira geral e especificamente confrontando as noções de desenvolvimento sustentável e ecoturismo; (4) abarcam os discursos dicotômicos das relações entre ambiente e cultura, no campo das ações construídas socialmente, bem como, (5) necessitam ganhar significado para a sociedade e a Educação Ambiental, enquanto uma prática social pode contribuir para tal se fomentar a integração participativa e democrática nas decisões sobre a gestão dos Parques.

Porém, esses mesmos autores ressaltam que a há uma visão fragmentada sobre a aplicação dos preceitos da educação ambiental em parques, ora relacionada ao fomento da capacitação comunitária para a sua gestão democrática, ora centrada na mitigação de impactos da visitação. Pragmaticamente, o resultado é que a educação ambiental geralmente não acontece nos parques a partir da vontade e planejamento gerencial, apesar do PRONEA (MMA, 2005, p.34) considerar que a educação ambiental é "[...] um dos instrumentos fundamentais da gestão ambiental" ressaltando que o Programa almeja "ressaltar o bom exemplo das práticas e experiências exitosas, como a integração entre professores e técnicos ambientais em programas de formação."

Logo, considerando que os parques são os inerentes receptores de atividades de educação ambiental, a gestão depende do estabelecimento de parcerias com escolas e organizações não governamentais para que elas aconteçam, pois a emergência das questões do uso público não permite a construção de um processo de ensino mais aprofundado. Nesses casos, os gestores geralmente optam pela interpretação ambiental, que está relacionada com as experiências subjetivas no ambiente natural (VASCONCELLOS, 2006), focando na explicação dos fundamentos técnicos que baseiam as restrições impostas para a mitigação dos impactos da visitação.

No âmbito dos acordos internacionais, a educação ambiental para as comunidades do entorno e visitantes, configura-se como uma importante estratégia de ação prevista na Convenção da Diversidade Biológica (CDB) e deve ser traduzida em políticas públicas nacionais. Ainda, frente à necessidade desenvolvimento de metodologias, recentemente foi aprovada a "Estratégia Nacional de Comunicação e Educação Ambiental", proposta pelo Ministério do Meio Ambiente (MMA, 2012). Nesse sentido, a universidade tem o papel fundamental na pesquisa e desenvolvimento das atividades de educação ambiental em unidades de conservação para que essas diretrizes políticas sejam efetivadas.

\section{Monitoramento de impactos}

Como já observado, o uso público dos parques causa impactos ambientais, que precisam ser manejados. No caso das áreas de uso mais intenso, o manejo apoia-se no conceito de capacidade de carga. Essa pode ser definida como o nível máximo de uso que uma área pode sustentar frente à alteração de fatores físicos, sociais, institucionais e ecológicos, que apresentam limites além dos quais o uso deve ser reduzido (MAGRO, 1999; MANING, 1986; STANKEY, MCCOOL, STOKES, 1990). Esse conceito é bastante 
complexo e recebeu muitas críticas quando passou a ser interpretado de maneira estreita, focando primordialmente no número de visitantes que uma determinada área poderia suportar (CIFUENTES, 1993). Porém, como coloca Hammitt e Cole (1998), não há relação linear desse parâmetro com os impactos causados, o que aumenta a complexidade da sua aplicação prática e sublinha as diferenças comportamentais dos visitantes. Atualmente, a palavra chave do manejo do uso público refere-se ao monitoramento dos impactos, pelo estabelecimento de uma estrutura de tomada de decisões baseada em indicadores e padrões (NILSEN, TAYLER, 1998).

Uma variedade de métodos abrange essa conceituação mais ampla, como o LAC ("Limits of Accetable Change" - STANKEY et al., 1985), o VIM ("Visitors Impact Management" - GRAEFE, KUSS, VASKE, 1990), o VAMP (Visitors Activity Management Process - GRAHAM, 1989, apud TAKAHASHI, 2004) e o VERP (Visitors Experience and Resource Process - MANNING, et al., 1995). Todos esses métodos envolvem procedimentos similares, pois conceitualmente estabelecem parâmetros de capacidade suporte de visitação em áreas naturais, protegidas ou não. Todos também enfatizam as condições do recurso para substanciar as decisões de manejo (PASSOLD, 2002).

Segundo Takahashi, Milano e Tormena (2005), a limitação dos recursos, equipamentos e pessoal frente ao desenvolvimento desordenado da visitação em unidades de conservação torna premente o desenvolvimento de métodos nacionais para a realização de avaliações sistemáticas de impactos do uso público. Nesse contexto, insere-se a universidade e o seu papel com a pesquisa, o ensino e a extensão na constituição de parcerias com os órgãos ambientais, para a realização de trabalhos periódicos de avaliação de impactos da visitação em parques.

\section{Aliando as atividades acadêmicas com a gestão do uso público em parques}

O processo de inserção social dos parques é basal para os esforços de conservação (PIMENTEL; MAGRO, 2012). Nesse sentido, duas premissas devem ser consideradas: a educação ambiental com foco na mudança de comportamento e o monitoramento dos impactos da visitação. Ambas estão relacionadas com o manejo do uso público nessas áreas protegidas. Assim, o Grupo de Estudos Interdisciplinares do Ambiente (GEIA DCIEN/FFP/UERJ) fomenta uma série de atividades que transcendem a pesquisa acadêmica básica, envolvendo alunos e professores universitários em projetos que também são enquadrados como atividades de ensino e extensão.

Considerando a avaliação de impactos decorrentes do uso público, desde 2009 o grupo realiza o monitoramento com frequência semestral, da trilha do Poço Verde no Parque Nacional da Serra dos Órgãos no estado do Rio de Janeiro (PIMENTEL; MAIA; BARROS, 2012). Esse monitoramento resulta de uma atividade de ensino, proposta na cadeira de Ecologia. A gestão do Parque tem aplicado os conhecimentos gerados na gestão do seu uso público, pois os indicadores utilizados dão respostas estatisticamente significativas. Há também congruência com as atividades de estagiários e monitores do GEIA com as demandas de gestão da visitação no Parque Estadual da Serra da Tiririca (Niterói, Maricá/RJ). Diferentes projetos de pesquisa e extensão são realizados nessa unidade de conservação e envolvem a 
definição de indicadores para avaliação de impactos do uso público na trilha do Bananal (PIMENTEL; MAIA; BARROS, 2012), formulação de trilhas interpretativas e mapeamento das mesmas.

No que concerne à educação ambiental, há atividades de excursões didáticas em trilhas desde 2008 (MEIRELES; PIMENTEL, 2008), bem como uma parceria formalmente instituída entre o GEIA e o Museu de Arqueologia de Itaipu (MAI - Niterói/RJ). O MAI possui um Programa Educativo e Cultural e já atendia crianças e comunidade em consonância com a sua política de visitação. No entanto, ainda carecia de uma aproximação maior com a comunidade local. Essa tem sido viabilizada por um projeto interinstitucional que também envolve o parque Estadual da Serra da Tiririca e escolas da região, permitindo o desenvolvimento de atividades ao longo do ano (MATTA; PIMENTEL; FERREIRA, 2011). Nesse sentido, o GEIA foi contemplado com verbas em editais de financiamento de pesquisa da FAPERJ, inclusive para a produção de material didático direcionado a esse projeto. Outra ação importante do grupo de pesquisa foi uma avaliação diagnóstica das atividades de educação ambiental em parques do estado do Rio de Janeiro (SANTOS, 2013).

\section{Considerações finais}

Frente aos conceitos e experiências apresentadas podem-se apontar as vantagens do estabelecimento de parcerias entre os gestores do uso público de parques e a universidade, pois há possibilidades de se: (1) Aliar as atividades acadêmicas de ensino, pesquisa e extensão às demandas da gestão da visitação; (2) Superar os desafios impostos pelas fragilidades financeiras e administrativas dos órgãos gestores e do financiamento de pesquisa; (3) Capacitar reciprocamente gestores e alunos; (4) Selecionar indicadores avaliativos, de utilização factível pelos alunos e pela gestão, devido à facilidade de treinamento e simplicidade metodológica; (4) Formar séries históricas de dados para o efetivo monitoramento; (5) Desenvolver as metodologias de monitoramento; (6) Aproximar as Instituições entre si e com as comunidades adjacentes aos parques; (7) Promover a inserção social dos parques; (8) Construir uma base metodológica e conceitual para a realização de educação ambiental em parques (9) Permitir ao órgão gestor, a realização dessas atividades e finalmente (10), Adequar a gestão às políticas nacionais de conservação e educação ambiental. Para tal é necessário que haja o efetivo exercício da interdisciplinaridade e a melhoraria da comunicação e troca de informações entre as instituições envolvidas para que uma verdadeira parceria seja desenvolvida.

\section{Referências bibliográficas}

ABAKERLI, S. A critique of development and conservation policies in environmentally sensitive regions in Brazil. Geoforum, Amsterdam, v.32, p. 551-556, 2001.

ARAUJO, G.P.; GELBCKE, D.L. Turismo Comunitário: uma perspectiva ética e educativa de desenvolvimento. Revista Turismo Visão e Ação - Eletrônica, v.10, n.3. p.357-378, 2008.

BENSUSAN, N. Conservação da biodiversidade em áreas protegidas. Rio de Janeiro: Ed. FGV, 2006. $176 \mathrm{p}$. 
BOO, E. O planejamento ecoturístico para áreas protegidas. In: LINDBERG, K.; HAWKINS, D.E. (Ed.). Ecoturismo: um guia para planejamento e gestão. 2. ed. São Paulo: Ed. SENAC, 1999. cap. 1, p. 31-57.

BRASIL. LEI No 9.985, de 18 de julho de 2000. Institui o Sistema Nacional de Unidades de Conservação da Natureza(SNUC). Brasília: IBAMA, Diretoria de Ecossistemas, 2002. 35 p.

BRASIL. Instrução Normativa No 154, de 01 de Março de 2007. Institui o Sistema de Autorização e Informação em Biodiversidade (Sisbio) e o Comitê de Assessoramento Técnico do Sistema de Autorização e Informação em Biodiversidade (CAT-Sisbio). Brasília: IBAMA. 2007.

CIFUENTES, M. Determinación de capacidad de carga turística em áreas protegidas. WWF/CATIE. Turrialba, Costa Rica. 1993. 26p.

DIAS, G.F. Educação ambiental: princípios e práticas. São Paulo: Ed. Gaia, 2003. 551 p.

DIEGUES, A.C. O mito moderno da natureza intocada. 5. ed. São Paulo: Hucitec; Núcleo de apoio à pesquisa sobre populações humanas e áreas úmidas brasileira.USP, 2004. 169 p.

GODOY, A. O modelo de natureza e a natureza do modelo. São Paulo em Perspectiva, São Paulo, v. 4, n. 14, p. 129-138, 2000.

GRAEFE, A.R.; KUSS, F.R.; VASKE, J.J. Visitor Impact Management. The Planning Framework. National Parks and Conservation Association, Washington, D.C., v.2. 1990. 105 p.

LOUREIRO, C.F.B. Educação ambiental e gestão participativa na explicitação e resolução de conflitos. Gestão em Ação, Salvador, v. 7, n. 1, p. 1-16, jan./abr. 2004.

MAGRO, T.C. Impactos do uso público em uma trilha no planalto do Parque Nacional de Itatiaia. 1999.135 f. Tese (Doutorado em Engenharia Florestal) - Escola de Engenharia de São Carlos, Universidade de São Paulo. São Carlos.

MAGRO, T. C. Percepções do Uso Público em UCs de Proteção Integral. In: Alex Barger. (Org.). Áreas Protegidas: Conservação no Âmbito do Cone Sul. Pelotas. Pelotas, v. 1, p. 8798, 2003.

MANING, R.E. Density, crowding and satisfaction: search for relationships. In: Studies in outdoor recreation. Ovvallis: Oregon State University press, 1986. p. 49-77. cap. 5.

MANNING, R.E.; LIME, D.W.; HOT, M. et al. The visitor experience and resource protection (VERP) process: the application of Carrying Capacity to Arche National Park. The George Wright Forum. v.12., n. 3, p. 41-55, 1995.

MATTA, R. F.; PIMENTEL, D. S.; FERREIRA, M. S.A Educação Ambiental como Mediadora na Diluição de Fronteiras Interinstitucionais. In: Giovanni Seabra; Ivo Mendonça. (Org.). Educação Ambiental: Responsabilidade para a Conservação da Sociobiodiversidade. 2. ed. .João Pessoa: Editora Universitária da UFPB , 2011. v. 4, p. 1461-1469.

MEIRELES, C. P.; PIMENTEL, D. S. A Educação Ambiental como mediadora na mudança de percepção sobre o Parque Estadual da Serra da Tiririca (Niterói/Marica - RJ). In I Enconto Científico do Parque Estadual da Serra da Tiririca. Anais... Universidade Federal Fluminense: Niterói-RJ. p.43-49, 2008.

MINISTÉRIO DO MEIO AMBIENTE. Programa Nacional de Educação Ambiental ProNEA. Diretoria de Educação Ambiental; Ministério da Educação. Coordenação Geral de Educação Ambiental. 3. ed. Brasília:MMA, 2005. 102 p. 
MINISTÉRIO DO MEIO AMBIENTE. Diretrizes para a Estratégia Nacional de Comunicação e Educação Ambiental em Unidades de Conservação. ICMBio. , 2012. Disponível

em: http://www.icmbio.gov.br/portal/images/stories/comunicacao/publicacao_encea.pdf. $>$. Acesso em: 3 maio 2013.

NATIONAL PARK SERVICE. Yellowstone National Park. Disponível em: <http://www.nps.gov/yell/historyculture/>. Acesso em: 02 Maio 2013.

NILSEN, P.; TAYLER, G. A Comparative Analysis of Protected Area Planning and Management Frameworks. In: McCool, Stephen F.; Cole, David N., comps. Proceedings Limits of Acceptable Change and related planning processes: progress and future directions; Missoula, MT. Gen. Tech. Rep. INT-GTR-371. Ogden, UT: U.S. Department of Agriculture, Forest Service, Rocky Mountain Research Station, 1998. p.49-58.

PASSOLD, A. J. Seleção de indicadores para o monitoramento do uso público em áreas naturais. 2002. 75 p. Dissertação (Mestrado em Recursos Florestais, com opção em Conservação de Ecossistemas Florestais) - Escola Superior de Agricultura "Luiz de Queiroz", Universidade de São Paulo, Piracicaba, 2002.

PIMENTEL, D.S.; MAGRO, T.C. The symbolic sphere and social representations of Serra da Tiririca State Park, Rio de Janeiro, Brazil. Sociedade \& Natureza, Uberlândia, ano 23, n. 2, p. 275-283, 2011.

PIMENTEL, D.S.; MAGRO, T. C. Múltiplos olhares, muitas imagens: o manejo de parques com base na complexidade social. GEOgraphia (UFF). , v. 2011, p. 92 - 113, 2012.

PIMENTEL, D.S.; MAGRO, T.C. Diferentes Dimensões da Educação Ambiental para a Inserção Social dos Parques. Revista Brasileira de Educação Ambiental, Rio Grande, Número Especial, v. 2012, p. 44-50. No prelo.

PIMENTEL, D. S.; MAIA, S.; BARROS, A.A.M. A extensão universitária na trilha do uso público em parques. Interagir (UERJ), v. 2010, p. 95-99, 2012.

SANTOS, D.C.R. Diagnóstico das ações de Educação Ambiental em Parques Estaduais do Rio de Janeiro. 2013. 48p. Trabalho de Conclusão de Curso (Licenciatura em Ciências Biológicas). Faculdade de Formação de Professores - Universidade do Estado do Rio de Janeiro. 2013.

SILVA, N.P.S.; COSTA NETO A.R. A educação ambiental como instrumento de sensibilização turística em unidades de conservação. 3. ed. Revista Eletrônica Aboré, Manaus, 2007. Disponível em: $<\mathrm{http}$ ///www.revista.uea.edu.br/abore/comunicacao/comunicacao_pesq_3/Nathalin\%20Priscil a\%20de\%20Souza\%20da\%20Silva.pdf>. Acesso em: 27 mar. 2008.

STANKEY, G.H.; COLE, D.N.; LUCAS, R.C et al. The limits of acceptable change (LAC) system for wilderness planning. General Technical Report INT-176. Ogden, UT. USDA Forest Service Intermountain Forest and Range Experiment Station, 1985. 37p.

STANKEY, G. H., MCCOOL, S. F.; STOKES, G. L. Managing for appropriate wilderness conditions: The carrying capacity issue. In J. C. Hendee \& G. H. Stankey \& R. C. Lucas (Ed,.), Wilderness Management. 2.ed. 1990. p. 215-239.

TAKAHASHI, L. Uso público em unidades de conservação. Cadernos de Conservação, Curitiba, v. 2, n. 2, out. 2004. 40 p. 
TAKAHASHI, L.Y.; MILANO, M.S.; TORMENA, C.A. Indicadores de impacto para monitorar o uso público no Parque Estadual Pico do Marumbi, Paraná. Revista Árvore. v.29, n.1, p.159-167, 2005.

UNESCO. Educação ambiental: as grandes orientações da Conferência de Tbilisi. Brasília: Instituto Brasileiro de Meio Ambiente e Recursos Naturais Renováveis, 1998. 154 p.

VASCONCELLOS, J.M. Educação e interpretação ambiental em unidades de conservação. Cadernos de Conservação, Curitiba, v. 3, n. 4, 86 p. dez. 2006.

WATSON, A.E. Wilderness use in the year 2000: societal changes that influence human relationships with wilderness. USDA forest service proceedings RMRS, v.4, p.53-60, 2000.

WATSON, A.E.; LANDRES, P. Changing wilderness values. In: CORDELL, H. K. (Org.). Outdoor recreation in American life: a national assessment of demand and supply trends. Champaign, IL: Sagamore, 1999. p.384-388.

WEST, P.; IGOE, J.; BROCKINGTON, D. Parks and people: the social impact of protected areas. Annual Review of anthropology, Palo Alto, v. 35, p. 251-277, 2006.

WESTERN, D. Prefácio: definindo ecoturismo. In: LINDBERG, K.; HAWKINS, D.E. (Ed.). Ecoturismo: um guia para planejamento e gestão. 2. ed. São Paulo: Ed. SENAC, 1999. p.1322. 